\title{
Do Upper Extremity Trauma Patients Have Different Preferences for Shared Decision-making Than Patients With Nontraumatic Conditions?
}

\author{
Michiel G. J. S. Hageman MD, Rajesh Reddy BA, Dennis J. S. Makarawung BSc, \\ Jan Paul Briet MD, C. Niek van Dijk MD, PhD, David Ring MD, PhD
}

Published online: 4 June 2015

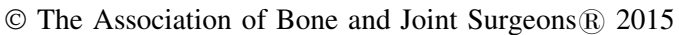

\begin{abstract}
Background Shared decision-making is a combination of expertise, available scientific evidence, and the preferences of the patient and surgeon. Some surgeons contend that patients are less capable of participating in decisions about traumatic conditions than nontraumatic conditions.

Questions/purposes (1) Do patients with nontraumatic conditions have different preferences for shared decisionmaking when compared with those who sustained acute trauma? (2) Do disability, symptoms of depression, and self-efficacy correlate with preference for shared decisionmaking?

Methods In this prospective, comparative trial, we evaluated a total of 133 patients presenting to the outpatient practices of two university-based hand surgeons with traumatic or nontraumatic hand and upper extremity illnesses or conditions. Each patient completed questionnaires
\end{abstract}

One of the authors (MGJSH) is supported by Dutch research grants from the Marti-Keunig Eckhart Stichting and Anna Foundation. All ICMJE Conflict of Interest Forms for authors and Clinical Orthopaedics and Related Research ${ }^{\circledR}$ editors and board members are on file with the publication and can be viewed on request.

Each author certifies that his or her institution approved the human protocol for this investigation, that all investigations were conducted in conformity with ethical principles of research, and that informed consent for participation in the study was obtained.

This work was performed at Massachusetts General Hospital, Boston, MA, USA.

M. G. J. S. Hageman, R. Reddy, D. J. S. Makarawung, J. P. Briet, D. Ring $(\bowtie)$

Orthopaedic Hand and Upper Extremity Service, Massachusetts General Hospital, 55 Fruit Street, Boston, MA 02114, USA

e-mail: dring@mgh.harvard.edu; dring@partners.org

C. N. van Dijk

Academic Medical Center, Amsterdam, The Netherlands measuring their preferred role in healthcare decision-making (Control Preferences Scale [CPS]), symptoms of depression (Patients' Health Questionnaire), and pain self-efficacy (confidence that one can achieve one's goals despite pain; measured using the Pain Self-efficacy Questionnaire). Patients also completed a short version of the Disabilities of the Arm, Shoulder, and Hand questionnaire and an ordinal rating of pain intensity.

Results There was no difference in decision-making preferences between patients with traumatic (CPS: $3 \pm 2$ ) and nontraumatic conditions (CPS: $3 \pm 1$ mean difference $=0.2$ [95\% confidence interval, -0.4 to 0.7$], \mathrm{p}=0.78$ ) with most patients (95 versus 38) preferring shared decisionmaking. More educated patients preferred a more active role in decision-making (beta $=-0.1, \mathrm{r}=0.08, \mathrm{p}=0.001$ ); however, differences in levels of disability, pain and function, depression, and pain-related self-efficacy were not associated with differences in patients' preferences in terms of shared decision-making.

Conclusions Patients who sustained trauma have on average the same preference for shared decision-making compared with patients who sustained no trauma. Now that we know the findings of this study, clinicians might be motivated to share their expertise about the treatment options, potential outcomes, benefits, and harms with the patient and to discuss their preference as well in a semiacute setting, resulting in a shared decision.

\section{Introduction}

In shared decision-making the caregiver provides expertise and evidence, and the patient and caregiver choose diagnostic and treatment options consistent with their values 
and preferences [23]. There is evidence that empowering patients to participate in decision-making with the help of decision aids (videos, web sites, or pamphlets that help patients understand their options and become aware of their preferences) results in increased satisfaction and physical function and reduced decisional conflict, anxiety, and resource utilization [22]. Patient preferences for involvement in decision-making may vary by age, sex, socioeconomic status, type of illness, and illness behavior, and perhaps the gravity or acuity of the decision [3, 17].

Many surgeons hold the opinion that patients with traumatic problems are less capable of and less interested in participating in decisions because they feel vulnerable and time-pressured. Although to our knowledge this has not been studied, many of our colleagues insist that patients with a painful acute fracture cannot fully participate in the decision-making process and need the doctor to recommend treatment. In addition, patients with greater symptoms of depression or less self-efficacy might have less desire or confidence about participation in the decision-making process and might prefer to fall back to a paternalistic style of medical care and take a more passive role. Depressed mood and ineffective coping strategies can make people feel more resigned, passive, and helpless. We therefore wished to assess hand surgery patient preferences for shared decisionmaking in relation to the acuity of the diagnosis and to psychological factors.

This study tested the following hypotheses: (1) Do patients with nontraumatic conditions have different preferences for shared decision-making when compared with those who sustained acute trauma? (2) Do disability, symptoms of depression, and self-efficacy correlate with preference for shared decision-making?

\section{Patients and Methods}

After approval from our institutional research board, all new, nonpregnant, English-speaking patients 18 years or older presenting to one of two hand surgeons (DR, CM) were asked to participate in this prospective study. The researcher informed the patient about the study details and informed consent was obtained. Patients were enrolled between November 2012 and April 2013.

We asked 135 patients to participate in the study: one $(0.7 \%)$ declined and 134 were enrolled before seeing the treating physician. One patient was excluded from the study as a result of invalid answers on one of the questionnaires. The analyses were conducted on 133 patients (68 men and 65 women) with a mean age of $47 \pm 17$ years (range, 18-86 years). The demographics of trauma and nontrauma cohorts were comparable (Table 1). There was also no difference in levels of education comparing the trauma (mean, 16 years; range, 9-16 years) and nontrauma cohorts (mean, 15 years; range, 0-20 years; $\mathrm{p}=0.10$ ). Conditions categorized as traumatic included: fracture, laceration, sprain, tendon injury, and amputation. All other diagnoses were considered nontraumatic; examples included arthrosis, carpal tunnel, trigger finger, and another discrete diagnosis.

\section{Measurement Tools}

At the time of enrollment, patients completed a demographic survey, including level of education, and the following questionnaires: the Control Preferences Scale (CPS), the short version of the Disabilities of the Arm, Shoulder and Hand questionnaire (QuickDASH), the Pain Self-efficacy Questionnaire (PSEQ), the short version of the Patients Health Questionnaire (PHQ-2), and an 11-point ordinal pain intensity score. After the encounter with the physician, the research assistant registered whether the patient was a trauma or nontrauma patient.

Education, as the number of years of school, was measured on a continuous scale with graduation from high school scored as 12 .

The CPS is a validated measure of a patient's preferred role in healthcare decision-making [6]. Patients rank-order five possible approaches to decision-making, resulting in a score that is scaled from 1 (most active role) to 6 (most passive role). A score of 3 or lower indicates a preference for shared decision-making [6].

The QuickDASH is a short version of the DASH and is used to determine arm-specific disability [2, 10]. It consists of 11 questions, which are answered on a 5-point Likert scale. The total score is scaled to range from 0 (no disability) to 100 (most severe disability).

The PSEQ is a questionnaire designed to assess a patient's confidence that they can achieve their goals despite pain $[1,20]$. It involves 10 items, which can be scored by the patient on a 7-point Likert scale, ranging from 0 (not at all confident) to 6 (completely confident). The outcome score is calculated by adding up the items on a scale ranging from 0 to 70 . A higher score indicates greater confidence. Mean imputation was used for two missing values.

The PHQ-2 was used to evaluate symptoms of depression. The PHQ-2 is a validated two-question measure of symptoms of depression [15, 16]. The two questions are answered on a 4-point Likert scale ranging from 0 (not at all) to 3 (nearly everyday) and the overall score ranges from 0 to 6 .

The Numeric Rating Scale is an 11-point ordinal measure of pain intensity. 
Table 1. Demographics

\begin{tabular}{|c|c|c|c|c|}
\hline \multirow[t]{2}{*}{ Parameter } & \multicolumn{2}{|c|}{ Trauma cohort $(\mathrm{N}=67)$} & \multicolumn{2}{|c|}{ Nontrauma cohort $(\mathrm{N}=66)$} \\
\hline & Mean & Range & Mean & Range \\
\hline Age (years) & 45 & $18-86$ & 49 & $20-86$ \\
\hline \multirow[t]{2}{*}{ Education (years) } & 16 & $9-26$ & 15 & $0-20$ \\
\hline & Number & Percent & Number & Percent \\
\hline \multicolumn{5}{|l|}{ Sex } \\
\hline Women & 30 & 45 & 35 & 53 \\
\hline Men & 37 & 55 & 31 & 47 \\
\hline \multicolumn{5}{|l|}{ Marital status } \\
\hline Single & 32 & 48 & 20 & 30 \\
\hline Living with partner & 1 & 2 & 3 & 4.6 \\
\hline Married & 24 & 36 & 32 & 48 \\
\hline Separated/divorced & 5 & 8 & 6 & 9 \\
\hline Widowed & 4 & 6 & 5 & 8 \\
\hline \multicolumn{5}{|l|}{ Work status } \\
\hline Working, full-time & 42 & 63 & 40 & 61 \\
\hline Working, part-time & 5 & 8 & 6 & 9 \\
\hline Homemaker & 0 & 0 & 2 & 3 \\
\hline Retired & 7 & 10 & 11 & 17 \\
\hline Unemployed and able to work & 3 & 5 & 4 & 6 \\
\hline Unemployed and unable to work & 4 & 6 & 2 & 3 \\
\hline On workers compensation & 2 & 3 & 1 & 2 \\
\hline Currently on sick leave & 4 & 6 & 0 & 0.0 \\
\hline \multicolumn{5}{|l|}{ Diagnosis } \\
\hline Acute injury & 67 & 100 & & \\
\hline Nonspecific arm pain & & & 11 & 17 \\
\hline Trigger finger & & & 8 & 12 \\
\hline Carpal tunnel syndrome & & & 9 & 14 \\
\hline Ganglion cyst & & & 4 & 6 \\
\hline Arthrose & & & 11 & 17 \\
\hline Dequervain's & & & 3 & 5 \\
\hline Dupuytren & & & 4 & 6 \\
\hline Epicondylitis & & & 3 & 5 \\
\hline Bursitis & & & 1 & 1 \\
\hline Giant cell tumor & & & 1 & 1 \\
\hline Cubital tunnel syndrome & & & 2 & 3 \\
\hline Other & & & 9 & 14 \\
\hline \multicolumn{5}{|l|}{ Physician } \\
\hline Physician I & 17 & 25 & 24 & 36 \\
\hline Physician II & 50 & 75 & 42 & 64 \\
\hline Health outcomes & Mean & Range & Mean & Range \\
\hline QuickDASH & 43 & $2.3-86$ & 31 & $0-80$ \\
\hline Pain & 4.5 & $3.9-5.1$ & 5.4 & $4.7-6.0$ \\
\hline PSEQ & 42 & $2-60$ & 47 & $0-60$ \\
\hline PHQ & 1.4 & $0-6$ & 1 & $0-6$ \\
\hline
\end{tabular}

DASH = Disabilities of the Arm, Shoulder and Hand; PSEQ = Pain Self-efficacy Questionnaire; PHQ = Patients' Health Questionnaire. 
Table 2. Bivariable analyses

\begin{tabular}{|c|c|c|c|}
\hline \multirow[t]{2}{*}{ Parameter } & \multicolumn{2}{|c|}{ Control Preference Scale } & \multirow[t]{2}{*}{$\mathrm{p}$ value } \\
\hline & Mean & SD & \\
\hline \multicolumn{4}{|l|}{ Nonelective versus elective patients } \\
\hline Trauma cohort & 3 & 2 & \multirow[t]{2}{*}{0.78} \\
\hline Nontrauma cohort & 3 & 1 & \\
\hline \multicolumn{4}{|l|}{ Sex } \\
\hline Women & 3 & 2 & \multirow[t]{2}{*}{0.96} \\
\hline Men & 3 & 2 & \\
\hline \multicolumn{4}{|l|}{ Marital status } \\
\hline Single & 3 & 2 & \multirow[t]{5}{*}{0.39} \\
\hline Living with partner & 2 & 1 & \\
\hline Married & 3 & 2 & \\
\hline Separated/divorced & 4 & 2 & \\
\hline Widowed & 3 & 1 & \\
\hline \multicolumn{4}{|l|}{ Work status } \\
\hline Working, full-time & 3 & 2 & \multirow[t]{8}{*}{0.27} \\
\hline Working, part-time & 3 & 2 & \\
\hline Homemaker & 5 & 2 & \\
\hline Retired & 3 & 2 & \\
\hline Unemployed and able to work & 3 & 2 & \\
\hline Unemployed and unable to work & 3 & 2 & \\
\hline On workers compensation & 5 & 2 & \\
\hline Currently on sick leave & 3 & 2 & \\
\hline \multicolumn{4}{|l|}{ Diagnosis } \\
\hline Acute injury & 3 & 2 & \multirow{13}{*}{0.62} \\
\hline Nonspecific arm pain & 3 & 2 & \\
\hline Trigger finger & 4 & 1 & \\
\hline Carpal tunnel syndrome & 2 & 2 & \\
\hline Ganglion cyst & 4 & 2 & \\
\hline Arthrose & 3 & 2 & \\
\hline Dequervain's & 2 & 1 & \\
\hline Dupuytren & 2 & 1 & \\
\hline Epicondylitis & 2 & 1 & \\
\hline Bursitis & 3 & - & \\
\hline Giant cell tumor & 6 & - & \\
\hline Cubital tunnel syndrome & 3 & 1 & \\
\hline Other & 3 & 1 & \\
\hline \multicolumn{4}{|l|}{ Physician } \\
\hline Physician I & 3 & 1.6 & \multirow[t]{2}{*}{0.78} \\
\hline Physician II & 3 & 1.5 & \\
\hline Health outcomes & \multicolumn{2}{|c|}{ Coefficient } & $\mathrm{p}$ value \\
\hline Age & \multicolumn{2}{|c|}{-0.03} & 0.69 \\
\hline Education & \multicolumn{2}{|c|}{-0.27} & $<0.01$ \\
\hline Duration of injury & \multicolumn{2}{|c|}{-0.004} & 0.96 \\
\hline QuickDASH & \multicolumn{2}{|c|}{0.15} & 0.08 \\
\hline Pain & \multicolumn{2}{|c|}{0.082} & 0.35 \\
\hline PSEQ & \multicolumn{2}{|c|}{-0.1} & 0.24 \\
\hline
\end{tabular}


Table 2. continued

\begin{tabular}{lcc}
\hline Health outcomes & Coefficient & $p$ value \\
\hline PHQ & 0.00 & 0.96 \\
\hline
\end{tabular}

DASH = Disabilities of the Arm, Shoulder and Hand; PSEQ = Pain Self-efficacy Questionnaire; PHQ = Patients' Health Questionnaire.

\section{Statistical Analysis}

An a priori power analysis for our primary study question determined that 64 patients in the trauma cohort and 64 patients in the nontrauma cohort would provide $80 \%$ power to detect a $0.50 \mathrm{SD}$ (medium) difference in average CPS score with $\alpha=0.05$ using a two-tailed Student's t-test. We enrolled 135 patients to have at least 64 patients for each cohort accounting for dropouts and incomplete questionnaires.

In bivariate analysis, Pearson's correlation was used for continuous variables. The strength of a correlation between 0.10 to $0.29,0.30$ to 0.49 , and 0.50 to 1.0 is interpreted as small, medium, and large correlation, respectively [4]. The Student's t-test was used for the CPS (ordinal variable) when comparing between two groups; and analysis of variance was used to compare differences in CPS (again, ordinal variable) when more than two groups were present such as based on marital status. Variables with $\mathrm{p}<0.10$ were inserted in a backward, stepwise, multivariable linear regression analysis of factors associated with CPS. When categorical variables were inserted in multivariable analysis, dummy codes were generated when there were more than two categories.

\section{Results}

There was no difference between trauma (mean CPS: 3; SD: 2) and nontrauma patients' (mean CPS: 3; SD: 1) preferred level of shared decision-making (mean difference $=0.2$ [95\% confidence interval, -0.4 to 0.7$], \mathrm{p}=0.78$; Table 2). Again, scores of 3 or lower on the CPS suggest a desire on the part of the patient to engage in shared decision-making.

More educated patients had a greater desire to participate in decision-making (coefficient $=-0.27, \mathrm{p}<0.01$ ); but age, duration of complaint, disability level, pain or pain selfefficacy, and symptoms of depression were not associated with preferences for shared decision-making (Table 2).

\section{Discussion}

Assuming that patients with acute injury are less interested or capable of participating in decision-making risks devaluing their preferences. We found that patients with acute hand and upper extremity trauma prefer to be as engaged in decision-making as patients with nontraumatic conditions. As education levels increased, patients' desires to participate in shared decision-making also increased, which is consistent with prior research [11, 12, 24]. Coping strategies and symptoms of depression did not affect decision-making preferences.

This study should be considered in light of its shortcomings. First, the setting was limited to hand and upper extremity conditions. These findings may only generalize to other conditions or other practice settings, but that seems unlikely. It is possible that for some specific conditions, however, such as very severe trauma, the findings would be different. On the other hand, the lack of correlation between the duration since injury and the CPS suggests that time pressure does not have a strong influence.

Patients have similar levels of desire for shared decision-making, regardless of whether the condition was traumatic or nontraumatic. Decision-making preferences were addressed in a study of Korean patients with carpal tunnel syndrome [9]. Thirty-three percent of patients felt less involved in the decision-making regarding carpal tunnel release than they desired. Seventy-six percent of patients who preferred shared decision-making had lower scores on the DASH questionnaire compared with those who preferred a fully active or fully passive role [9]. There is some evidence that decision aids can help patients achieve their preferred role in decision-making [14, 19]. In general, patients who actively contribute to their health care have better functional outcome, choose less invasive treatments, and are more satisfied with their options $[5,8$, $11,18]$. Patients' outcomes and their satisfaction seem to be enhanced by higher levels of patient engagement. Providing patients with their desired level of involvement in decision-making is an important part of improving patient engagement and clinical results.

It may be surprising that the magnitude of education is the only factor associated with the desire to participate in shared decision-making and that age, duration of complaint, magnitude of disability level, pain intensity, and psychological factors did not have a measurable influence. There is a bias that shared decision-making is more acceptable to younger patients [7], but the finding that age is not associated with preferences for participation in the 
decision-making process agrees with prior studies [3]. Furthermore, one might guess that depressed mood and ineffective coping strategies might make people feel more resigned, passive, and helpless; our findings suggest that these factors do not influence preferences for participation in the decision-making process.

Many surgeons are of the opinion that injured patients must rely and prefer to rely on the surgeon's advice and feel less capable of participating in decision-making (as a result of pain, limited time to decide, etc) than patients with nontraumatic problems. One might also assume that older patients prefer a more paternalistic style and that patients with greater stress, distress, and less effective coping strategies will be more passive. This study in combination with prior studies demonstrate that shared decision-making is preferred by both trauma and nontrauma patients without obvious differences between those two groups of patients $[13,21,25]$. Patients, regardless of their level of education, deserve to participate in shared decision-making, but to give less-well-educated patients the confidence to do so, appropriate tools need to be developed. A decision aid appropriate for low levels of health literacy might increase a less educated patient's confidence that they can participate in decision-making. In our opinion, it is safe to assume that all patients prefer to participate in decisionmaking unless they suggest otherwise. Surgeons should provide accurate, balanced, dispassionate information to patients so that they can understand their preferences. We believe that most surgeons would agree that, given the uncertainty about the best management of many problems, the preferences of the patient should feature prominently in decision-making. Future research should help determine the best way to inform patients so that they feel adequately involved in the decision-making process and surgeon-tosurgeon variation in management is minimized. We think decision aids hold promise for achieving these goals and plan to develop aids and test their impact on decisional conflict, surgeon-to-surgeon variation, satisfaction with patient care, symptoms, and disability.

Acknowledgments We thank Chaitanya Mudgal MD, for his support in this study by creating the possibility to enroll patients from his outpatient clinic.

\section{References}

1. Asghari A, Nicholas MK. Pain self-efficacy beliefs and pain behaviour. A prospective study. Pain. 2001;94:85-100.

2. Beaton DE, Wright JG, Katz JN. Development of the QuickDASH: comparison of three item-reduction approaches. $J$ Bone Joint Surg Am. 2005;87:1038-1046.

3. Charles C, Gafni A, Whelan T. Shared decision-making in the medical encounter: what does it mean? (or it takes at least two to tango). Soc Sci Med. 1997;44:681-692.
4. Cohen JW. Statistical Power Analysis for Behavioral Sciences. 2nd ed. Hillsdale, NJ, USA: Lawrence Erlbaum Associates; 1988:79-81.

5. Degner LF, Kristjanson LJ, Bowman D, Sloan JA, Carriere KC, O'Neil J, Bilodeau B, Watson P, Mueller B. Information needs and decisional preferences in women with breast cancer. JAMA. 1997;277:1485-1492.

6. Degner LF, Sloan JA, Venkatesh P. The Control Preferences Scale. Can J Nurs Res. 1997;29:21-43.

7. Frosch DL, Kaplan RM. Shared decision making in clinical medicine: past research and future directions. Am J Prev Med. 1999;17:285-294.

8. Golin C, DiMatteo MR, Duan N, Leake B, Gelberg L. Impoverished diabetic patients whose doctors facilitate their participation in medical decision making are more satisfied with their care. J Gen Intern Med. 2002;17:857-866.

9. Gong HS, Huh JK, Lee JH, Kim MB, Chung MS, Baek GH. Patients' preferred and retrospectively perceived levels of involvement during decision-making regarding carpal tunnel release. J Bone Joint Surg Am. 2011;93:1527-1533.

10. Gummesson C, Ward MM, Atroshi I. The shortened Disabilities of the Arm, Shoulder and Hand questionnaire (QuickDASH): validity and reliability based on responses within the full-length DASH. BMC Musculoskelet Disord. 2006;7:44.

11. Hack TF, Degner LF, Dyck DG. Relationship between preferences for decisional control and illness information among women with breast cancer: a quantitative and qualitative analysis. Soc Sci Med. 1994;39:279-289.

12. Hibbard JH, Cunningham PJ. How engaged are consumers in their health and health care, and why does it matter? Res Brief. 2008:1-9.

13. Hutchinson RH, Barrie JL. The effects of shared decision making in the conservative management of stable ankle fractures. Injury. 2015 Jan 17 [Epub ahead of print].

14. Kennedy AD, Sculpher MJ, Coulter A, Dwyer N, Rees M, Abrams KR, Horsley S, Cowley D, Kidson C, Kirwin C, Naish C, Stirrat G. Effects of decision aids for menorrhagia on treatment choices, health outcomes, and costs: a randomized controlled trial. JAMA. 2002;288:2701-2708.

15. Kroenke K, Spitzer RL, Williams JB. The PHQ-9: validity of a brief depression severity measure. J Gen Intern Med. 2001;16:606-613.

16. Kroenke K, Spitzer RL, Williams JB. The Patient Health Questionnaire-2: validity of a two-item depression screener. Med Care. 2003;41:1284-1292.

17. Légaré F. Establishing patient decision aids in primary care: update on the knowledge base. Z Evid Fortbild Qual Gesundhwes. 2008;102:427-430.

18. Légaré F, Stacey D, Briere N, Fraser K, Desroches S, Dumont S, Sales A, Puma C, Aube D. Healthcare providers' intentions to engage in an interprofessional approach to shared decisionmaking in home care programs: a mixed methods study. $J$ Interprof Care. 2013;27:214-222.

19. Murray E, Davis H, Tai SS, Coulter A, Gray A, Haines A. Randomised controlled trial of an interactive multimedia decision aid on benign prostatic hypertrophy in primary care. $B M J$. 2001;323:493-496.

20. Nicholas MK. The pain self-efficacy questionnaire: taking pain into account. Eur J Pain. 2007;11:153-163.

21. Slover J, Shue J, Koenig K. Shared decision-making in orthopaedic surgery. Clin Orthop Relat Res. 2012;470:1046-1053.

22. Stacey D, Bennett CL, Barry MJ, Col NF, Eden KB, HolmesRovner M, Llewellyn-Thomas H, Lyddiatt A, Legare F, Thomson R. Decision aids for people facing health treatment or screening decisions. Cochrane Database Syst Rev. 2011;10:CD001431.

23. Stiggelbout AM, Van der Weijden T, De Wit MP, Frosch D, Legare F, Montori VM, Trevena L, Elwyn G. Shared decision making: really putting patients at the centre of healthcare. $B M J$. 2012;344:e256. 
24. Uldry E, Schafer M, Saadi A, Rousson V, Demartines N. Patients' preferences on information and involvement in decision making for gastrointestinal surgery. World J Surg. 2013;37:2162-2171.
25. Youm J, Chenok KE, Belkora J, Chiu V, Bozic KJ. The emerging case for shared decision making in orthopaedics. Instr Course Lect. 2013;62:587-594. 